\title{
Analyse des hypoplasies dentaires associées au syndrome APECED : à propos de cinq cas
}

\author{
Analysis of enamel hypoplasia of APECED syndrome: report of five cases
}

\author{
JIHAD BENSID ${ }^{1}$, JEAN CHAUVÉ2 ${ }^{2}$ MICHEL POLAK ${ }^{3}$, SELIM BENNACEUR ${ }^{2}$, YVON ROCHE ${ }^{1}$, ISABELLE BAILLEUL-FORESTIER ${ }^{1}$
}

\section{RÉSUMÉ}

L'APECED (Autoimmune PolyEndocrinopathy-Candidiasis-Ectodermal Dystrophy) est une maladie héréditaire monogénique affectant les enfants en bas âge, à transmission autosomique récessive. Le gène, localisé sur le chromosome 21q22.3, est appelé gène AIRE (Auto-Immune REgulator). Le syndrome APECED est caractérisé par une candidose cutanéo-muqueuse chronique, de multiples endocrinopathies autoimmunes (hypoparathyroïdie, insuffisance surrénalienne, diabète...) et des dystrophies ectodermiques (vitiligo, alopécie, hypoplasie de l'émail dentaire...). Le but de cette étude est de décrire le phénotype dentaire de cinq patients atteints d'APECED et de rechercher une corrélation entre les signes bucco-dentaires de cette affection et son étiopathogénie. Une évaluation clinique et radiologique ainsi qu'une compilation des anomalies endocriniennes et systémiques associées ont été réalisées. Une hypoplasie de l'émail des dents permanentes est fréquente et elle peut même être un des premiers signes de la maladie, mettant en évidence le rôle de l'odontologiste. Med Buccale Chir Buccale 2007; 14 : 19-27.

mots clés: APECED, AIRE, candidose cutanéo-muqueuse chronique, polyendocrinopathie, dystrophie ectodermique, hypoplasie de l'émail dentaire

médecine buccale chirurgie buccale

VOL. $14, \mathrm{~N}^{\circ} 1$ 2008

page 19

\section{SUMMARY}

Autoimmune PolyEndocrinopathy-Candidiasis-Ectodermal Dystrophy (APECED) presents typically in childhood and is a monogenic autosomal disease with recessive inheritance. The defective gene responsible for this disease has been identified and named autoimmune regulator (AIRE). The AIRE gene is located on chromosome 21q22.3. APECED is characterized by chronic cutaneomucous candidiasis, multiple autoimmune endocrinopathies (hypoparathyroidism, adrenocortical failure, type 1 diabetes...) and ectodermal dystrophies (vitiligo, alopecia, dental enamel hypoplasia...). The aim of this study is to describe the dental phenotype of five patients with APECED and try to establish a correlation between the oro-dental defects and pathogeny of this syndrome. The study consisted in oral, clinical and radiological evaluations of these patients and compilation of endocrine and systemic defects associated. Enamel hypoplasia of permanent teeth is frequent and it can be the first sign of APECED, so dentist could have a role in diagnosis of this condition. Med Buccale Chir Buccale 2007; 13 : 19-27.

key words: APECED, AIRE, chronic cutaneomucous candidiasis, polyendocrinopathy, ectodermal dystrophy, enamel hypoplasia.

\footnotetext{
1. UFR d'Odontologie Université Paris 7 Service d'Odontologie Hôtel-Dieu Paris

2. Service de Chirurgie maxillo-faciale et Stomatologie Hôpital Robert Debré Paris

3. Service d'Endocrinologie pédiatrique Hôpital Necker Enfants Malades Paris
}

Demande de tirés à part:

Isabelle Bailleul-Forestier UFR d'Odontologie 5 rue Garancière 75006 Paris bailleul@ccr.jussieu.fr

Accepté pour publication le 21 janvier 2008 
médecine

buccale

chirurgie

buccale

VOL. $14, N^{\circ} 1$ 2008

page 20
Le spectre des manifestations cliniques du syndrome APECED est large : la candidose cutanéomuqueuse chronique, l'hypoparathyroïdie et la maladie d'Addison constituent la triade caractéristique de ce syndrome ${ }^{[1,2]}$. D'autres signes cliniques, bien que moins fréquents, peuvent aussi être associés. II s'agit d'endocrinopathies (hypogonadisme, diabète insulinodépendant, thyréopathie autoimmune), de maladies gastro- intestinales (atrophie gastrique, anémie de Biermer), d'une hépatite chronique et d'une dystrophie ectodermique (vitiligo, alopécie, altération de structure de l'émail dentaire, dystrophie des ongles). Les premières manifestations débutent dans l'enfance et l'évolution vers la polyendocrinopathie s'installe durant les deux premières décennies. Les autres signes peuvent se développer jusqu'à 50 ans. Dans la majorité des cas, la candidose constitue la première manifestation, survenant en général avant l'âge de 5 ans. Elle est généralement suivie d'une hypoparathyroïdie vers 10 ans, puis d'une insuffisance surrénalienne vers 15 ans. Ces trois manifestations majeures du syndrome surviennent selon cette chronologie, mais elles ne sont présentes, au moment du diagnostic, que dans un tiers des cas. La prévalence des manifestations augmente avec l'âge [3]. Cette affection est présente partout dans le monde, mais plus particulièrement dans certaines populations isolées en Finlande, en Sardaigne et en Iran. L'incidence est de 1/25000 pour les finlandais, 1/14000 pour les sardes et de 1/9000 pour les iraniens [4]. Au niveau biologique, le syndrome APECED se caractérise par la présence de divers auto-anticorps sériques spécifiques des tissus atteints. Leur mise en évidence constitue un élément important de diagnostic.

Le traitement du syndrome APECED inclut la gestion à long terme de l'infection candidosique et la correction des endocrinopathies présentes. Les déficits endocriniens sont en principe contrôlés par des thérapies substitutives conventionnelles. Le spectre très large de cette maladie implique un suivi régulier afin de détecter les nouvelles atteintes, certaines d'entre elles pouvant être létales [3]. Le tableau de dystrophie ectodermique du syndrome APECED peut comporter une atteinte des ongles et des membranes tympaniques, une alopécie, un vitiligo, une kératopathie et aussi une altération de structure de l'émail dentaire. En effet, selon Ahonen et coll. [1], $77 \%$ de ses 68 patients atteints du syndrome APECED présentent une altération de structure de l'émail des dents permanentes. Les observations retrouvées dans la littérature concernent une description médicale détaillée de la pathologie dans laquelle les manifestations dentaires sont citées (89 patients) ${ }^{[1,3]}$ et une présentation de quatre cas avec des lésions bucco-dentaires ${ }^{[5]}$; seules les dents permanentes sont atteintes.

Les anomalies dentaires sous forme d'hypoplasie de l'émail se traduisent par des aspects très variés, allant du simple défaut, punctiformes ou en bandes horizontales plus ou moins larges, jusqu'à des plages étendues dépourvues d'émail [6]. Les lésions suivent le modèle des périkymaties, en tournant horizontalement autour de la circonférence coronaire. La coloration varie du jaune au brun et il y a une alternance avec des zones d'émail bien formé. Le but de cette étude est de décrire le phénotype dentaire de patients atteints d'un syndrome APECED et de rechercher une corrélation entre les signes bucco-dentaires de cette affection et son étiopathogénie.

\section{MATERIEL ET METHODES}

Cinq patients, comprenant trois hommes et deux femmes, ayant un diagnostic génétique confirmé de syndrome APECED ont été inclus dans l'étude. Tous sont majeurs avec une distribution de l'âge s'étalant entre 18 et 41 ans.

L'anamnèse médicale a consisté en la compilation des anomalies endocriniennes et systémiques pour chaque patient. Puis un examen bucco-dentaire approfondi est venu compléter les données médicales. En premier lieu, une évaluation de l'indice carieux a été réalisée par le biais de l'indice CAO (nombre total de dents Cariées, Absentes, Obturées) basé selon les critères de l'Organisation Mondiale de la Santé. L'indice RI (Restorative Index $=\mathrm{O} / \mathrm{C}+\mathrm{O} x$ $100 \%$ ) a également été calculé pour les patients qui ont un indice $\mathrm{CAO} \neq 0$. Pour l'examen radiologique, seules les radiographies orthopantomographiques ont été obtenues ; les radiographies rétro-coronaires n'ont pu être réalisées. 
En second lieu, afin de quantifier les atteintes amélaires en rapport avec le syndrome APECED, leurs dents ont été examinées après séchage sous scialytique et les régions hypoplasiques explorées avec une sonde. II existe plusieurs méthodes pour classifier les dysplasies amélaires, et la plus classique est l'index DDE (Developmental Defects of Enamel). Celui-ci a été mis en place par la Fédération Dentaire Internationale ${ }^{[7]}$ afin de répertorier les différentes formes cliniques et de faciliter l'étude des dysplasies amélaires. Récemment, une nouvelle classification a été créée : l'EDI (Enamel Defects Index). Elle est plus reproductible, plus rapide et plus fiable que la précédente [8].

D'autres auteurs [9-11] ont eu une approche quantitative pour évaluer les hypoplasies de l'émail. Cette méthode est nommée « the hypoplastic area method » [12], et c'est celle-ci qui a été utilisé dans cette étude. Elle permet l'évaluation individuelle de la sévérité du défaut de chaque dent en mesurant la largeur des lésions hypoplasiques et en divisant cette valeur par la hauteur de la couronne. Cette valeur exprime la part de la surface coronaire impliquée dans la lésion. Si plusieurs lésions sont présentes sur la couronne, il est possible d'additionner les valeurs de chaque lésion afin d'obtenir la somme de la surface coronaire impliquée dans l'hypoplasie. Ce score final est appelé « Tooth Hypoplastic Area » (THA). Par exemple, une dent ayant des lésions occupant $50 \%$ de la surface coronaire a un THA de 0,5. Dans une population, le score de THA est calculé pour chaque paire de dent. Si une seule dent de la paire est présente, seul son THA est considéré. Ce score permet aux chercheurs d'établir la différence de susceptibilité aux agents responsables des défauts amélaires. De plus, la signification des valeurs de THA des dents d'un individu représente l'IHA («Individual Hypoplastic Area »). L'application de cette méthode utilisant les scores ("The hypoplastic area method ") est indubitablement difficile et souvent désagréable pour les patients. Cependant, elle est très utile pour la comparaison individuelle dans les syndromes rares.

\section{RESULTATS (Tab. 1, 2 et 3)}

Le Tableau 1 résume les atteintes médicales et bucco-dentaires des patients présentés.

La localisation précise des dents hypoplasiées par patient est détaillée :

Patient 1 (Fig. 1) :

- Hypoplasies sur les dents suivantes (Fig. 2 et 3) :

\begin{tabular}{lllll}
13 & 11 & 21 & 22 & 23 \\
\hline 43 & 41 & 31 & 32 & 33
\end{tabular}

Chez ce patient de 41 ans, c'est le bloc incisivocanin qui est touché. II a développé une hypoparathyroïdie à l'âge de 16 ans. Le diagnostic ayant été fait pendant l'adolescence, on peut supposer que la maladie n'était pas présente dans l'enfance, particulièrement lors de la période de minéralisation coronaire.

Patient 2 (Fig. 4) :

- Hypoplasies sur les dents suivantes (Fig. 5 et 6 ) :

\begin{tabular}{rrrrrr}
$18 \quad 16$ & 13 & 11 & 2122 & \multicolumn{2}{c}{26} \\
\hline $47 \quad 45$ & 4241 & 3132 & 35 & 38
\end{tabular}

Les hypoplasies de cette jeune femme de 24 ans (ayant des parents consanguins au premier degré) sont beaucoup plus importantes et concernent plus de dents que le patient 1 . Elle avait 4,5 ans lorsque l'hypoparathyroïdie a été diagnostiquée mais il est fort probable que les altérations du métabolisme phosphocalcique avaient débuté bien avant le diagnostic, pendant la période de minéralisation dentaire. Ce qui expliquerait certainement l'importance des hypoplasies observées.

Patient 3 (Fig. 7) :

- Hypoplasies sur les dents suivantes (Fig. 8 et 9) :

\begin{tabular}{ll}
13 & 23 \\
\hline 43 & 3334
\end{tabular}

II y a peu d'hypoplasies chez ce patient de 18 ans, petit frère du patient 2 . Toutes les canines sont atteintes ainsi que la première prémolaire mandibulaire gauche. II n'a pas encore développé d'hypoparathyroïdie.

Patient 4 (Fig. 10) :

Ce patient ne présente aucune hypoplasie malgré une hypoparathyroïdie diagnostiquée à l'âge de 11 ans.

Patient 5:

Cette patiente ne présente aucune hypoplasie malgré une hypoparathyroïdie diagnostiquée à l'âge de 3 ans. \begin{tabular}{l}
$\begin{array}{l}\text { médecine } \\
\text { buccale } \\
\text { chirurgie } \\
\text { buccale }\end{array}$ \\
\hline VOL. 14, N ${ }^{\circ} 1$ \\
2008
\end{tabular}

page 21 
médecine buccale chirurgie buccale

VOL. $14, N^{\circ} 1$ 2008 page 22

Tableau 1 : Résumé des manifestations médicales et bucco-dentaires. Summary of medical and oro-dental findings.

\begin{tabular}{|c|c|c|c|c|c|}
\hline & Patient 1 & Patient 2 & Patient 3 & Patient 4 & Patient 5 \\
\hline Age & 41 & 24 & 18 & 18 & 23 \\
\hline Candidose buccale & $+(1982)$ & + & + & + & $+(1996)$ \\
\hline Hypoparathyroïdie & $+(1982)$ & $+(1988)$ & - & $+(1992)$ & $+(1995)$ \\
\hline Insuffisance surrénalienne & $+(1982)$ & $+(1994)$ & $+(1997)$ & - & $+(1997)$ \\
\hline Insuffisance gonadique & - & $+(1995)$ & - & $+(2000)$ & - \\
\hline Diabète & - & - & - & - & - \\
\hline Hypothyroïdie & - & $+(1990)$ & - & - & - \\
\hline Hypoplasie amélaire & + & + & + & - & - \\
\hline Dystrophie unguéale & - & + & + & - & - \\
\hline Kératopathie & bilatérale & - & - & - & - \\
\hline Alopécie & - & $+(2002)$ & + & + & - \\
\hline Vitiligo & - & - & - & - & + \\
\hline Malabsorption intestinale & - & $+(1998)$ & - & - & $+(1998)$ \\
\hline Atteinte hépatique & - & $+(2002)$ & - & - & - \\
\hline Anémie de Biermer & - & $+(1995)$ & - & $+(2002)$ & - \\
\hline Atteinte pancréatique & - & $+(1998)$ & $+(1997)$ & - & - \\
\hline Traitement par vitamine D & Dédrogy $\left.\right|^{\circledR}$ & Uvédose ${ }^{\circledR}$ & Uvédose ${ }^{\circledR}$ & 0 & 0 \\
\hline Déficit en GH & - & - & $+(1997)$ & - & - \\
\hline Retard staturo-pondéral & - & - & $+[-2 \mathrm{DS}]$ & $+[-1 \mathrm{DS}]$ & $+[-1 \mathrm{DS}]$ \\
\hline Agénésie dentaire & - & $+23 \varnothing$ & $+12 \varnothing$ & - & - \\
\hline Hypoplasie amélaire & + & + & + & - & - \\
\hline Indice CAO & 10 & 17 & 18 & 0 & 0 \\
\hline Indice RI & 1 & 0,6 & 0,43 & 0 & 0 \\
\hline Indice IHA & 0,11 & 0,22 & 0,11 & 0 & 0 \\
\hline $\begin{array}{l}\text { +: symptôme présent } \\
\text { : symptôme absent }\end{array}$ & \multicolumn{5}{|c|}{$\begin{array}{l}\text { année de diagnostic } \\
\text { Déviation Standard }\end{array}$} \\
\hline
\end{tabular}

\section{DISCUSSION}

Les résultats concordent avec ceux de la littérature ${ }^{[1,3,5]}$. En effet, sur les cinq patients étudiés, deux patients ne présentent ni carie ni hypoplasie. Cependant, la moyenne des valeurs du THA observée par Perniola et coll. [5] $(0,418)$ est supérieure à celle des trois patients étudiés ayant des hypoplasies amélaires $(0,144)$ (Tab.2). Ceci peut être imputable à une sous-évaluation des altérations de notre part ou d'une atteinte moins importante de l'émail chez ces trois patients. En revanche, les résultats concordent avec ceux de Perniola et coll. [5] sur les dents les plus atteintes, à savoir les canines maxillaires. Mais dans les cas présentés, les incisives centrales maxillaires et les canines mandibulaires sont nettement moins atteintes que dans ceux de l'étude de Perniola et coll. [5] ; cette différence est encore plus marquée pour les prémolaires maxillaires où il n'y a pas d'hypoplasie. 


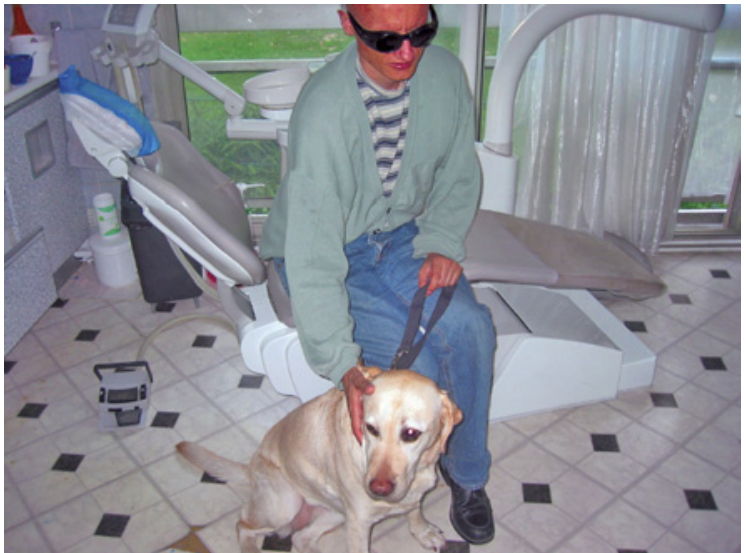

Figure 1 : Patient 1 ayant une cécité totale. Patient 1 affected by a complete blindness.

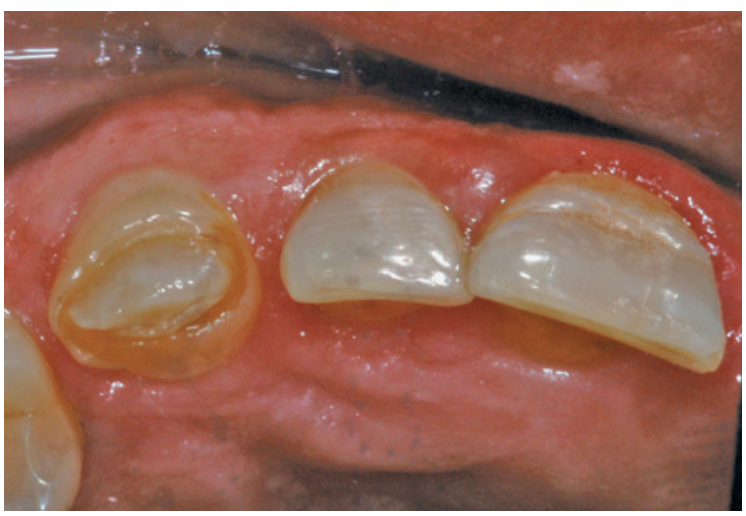

Figure $3: 11,12$ et 13 en vue occlusale (patient 1 ). 11, 12 and 13: occlusal view (patient 1).

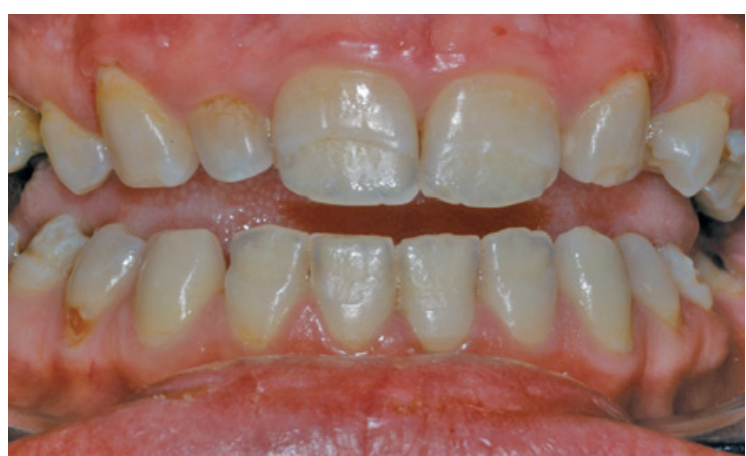

Figure 5 : Vue intra-buccale (patient 2). Intra-oral picture (patient 2).

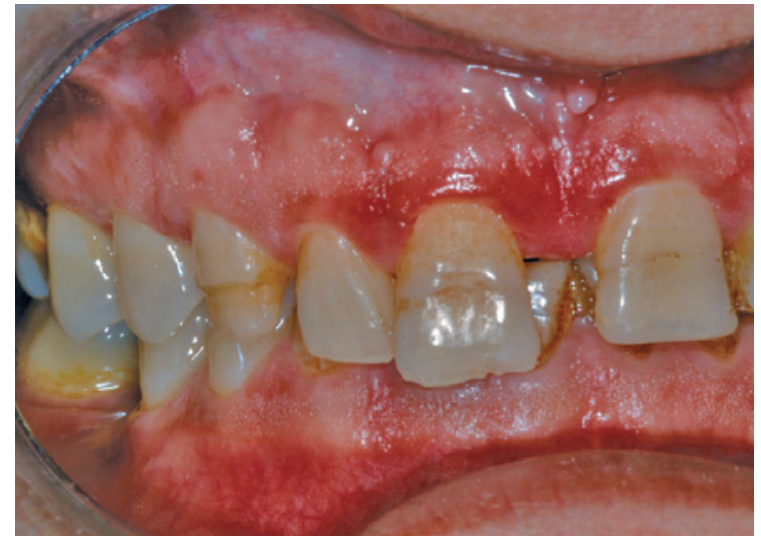

Figure 2 : Vue intra-buccale (patient 1). Intra-oral picture (patient 1).

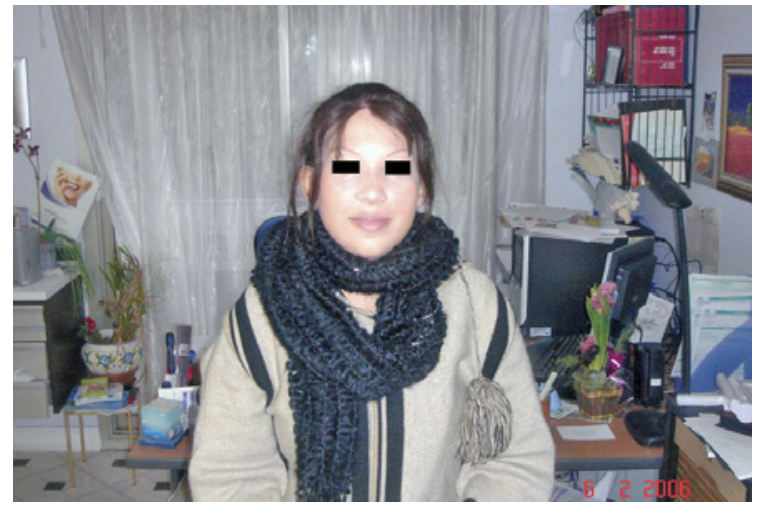

médecine

buccale

chirurgie

buccale

VOL. $14, N^{\circ} 1$ 2008

page 23
Figure 4 : Patient 2.

Patient 2.

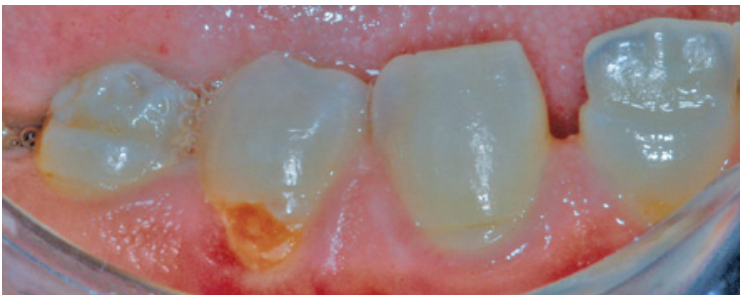

Figure 6 : Vue intéressant 42-45 (patient 2). Teeth 42-45 (patient 2). 


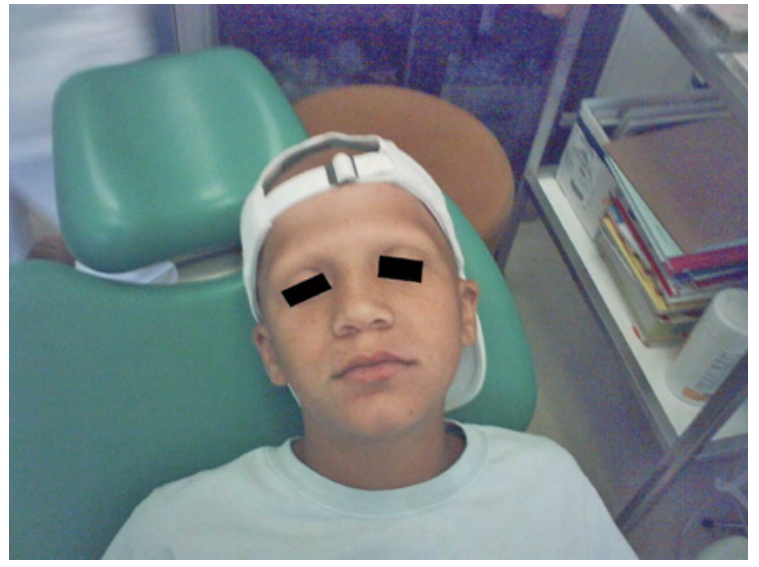

Figure 7 : Patient 3.

Patient 3.

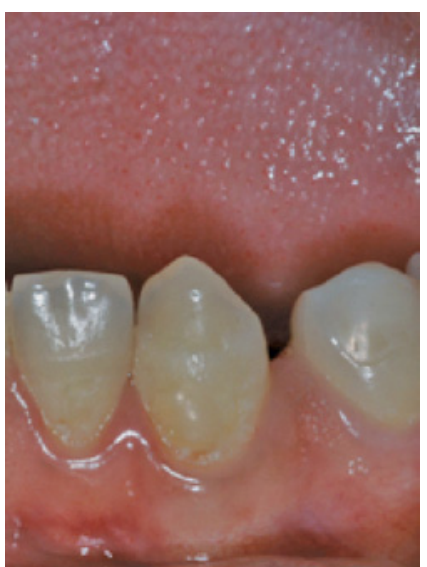

Figure 9 : Hypoplasie sur 33 et 34 (patient 3). Hypoplasia on 33 and 34 (patient 3).

Concernant I'IHA, les valeurs de Perniola et coll. [5] varient de façon plus importante (entre 0,11 et 0,6) alors que ces valeurs sont presque similaires pour les trois patients étudiés (Tab. 3). Quant à l'indice CAO des patients souffrant d'un syndrome APECED, il est nettement supérieur à la moyenne nationale qui est légèrement inférieure à 2 [13]. Cependant, cette moyenne correspond à l'indice CAO d'une population âgée de 12 ans ; or nos 3 patients sont nettement plus âgés, ce qui expliquerait, entre autres, l'indice plus élevé et la difficulté de comparer. Mais ils ont tout de même une hygiène très moyenne, voire médiocre. De plus, comme le montre l'indice RI, deux patients sur trois ne finissent pas leurs soins (Tab.1).

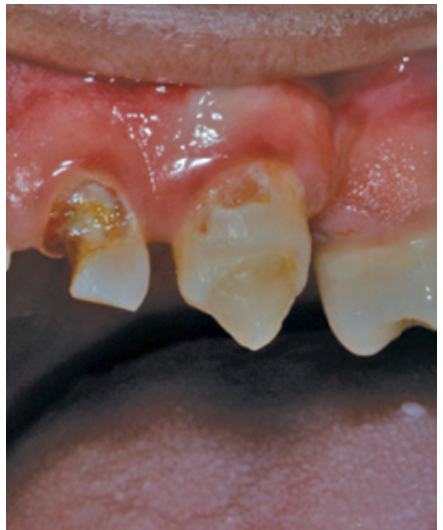

Figure 8 : Hypoplasie et carie sur 23 ; carie sur 22 (patient 3).

Hypoplasia and decay on 23 and decay on 22 (patient 3).

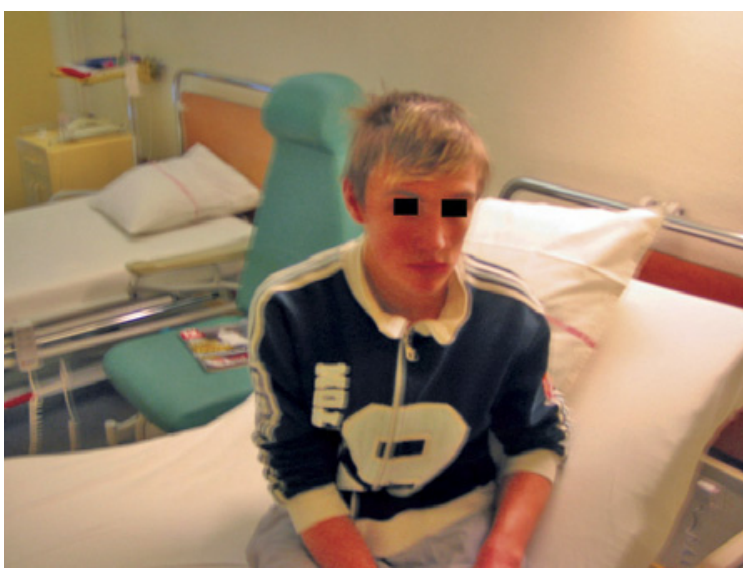

Figure 10 : Patient 4

Patient 4.

L'étude montre que les hypoplasies ne s'observent pas dans toutes les zones d'émail se formant à la même période. Plusieurs hypothèses pourraient expliquer ce phénomène.

Tout d'abord, la théorie des champs morphogénétiques. Selon Thomas et Sharpe ${ }^{[14]}$, il existe trois champs : incisif, canin et molaire. Ces auteurs proposent un homéocode dentaire déterminant une identité de forme et de position pour chaque entité dentaire. Cet homéocode suggère que pour chaque champ, il existe une combinaison spécifique de facteurs de transcription des gènes responsables de la morphologie des germes dentaires, dans l'espace et dans le temps. La formation de l'émail dentaire serait sous régulation 
Tableau 2 : Comparaison du THA (Tooth Hypoplastic Area) pour chaque type de dent dans cette étude et dans celle de Perniola et coll. [5].

THA (Tooth Hypoplastic Area) for each tooth type compared to Perniola's et coll. study ${ }^{[5]}$.

\begin{tabular}{ccc} 
Dent & THA & THA ${ }^{[5]}$ \\
\hline Maxillaire & & \\
Incisive centrale & 0,18 & 0,48 \\
\hline Incisive latérale & 0,07 & 0,35 \\
Canine & 0,42 & 0,56 \\
$1^{\text {re }}$ prémolaire & 0 & 0,40 \\
$2^{\circ}$ prémolaire & 0 & 0,47 \\
\hline Mandibulaire & & \\
Incisive centrale & 0,2 & 0,35 \\
Incisive latérale & 0,18 & 0,51 \\
Canine & 0,22 & 0,52 \\
$1^{\text {re }}$ prémolaire & 0,02 & 0,34 \\
$2^{\circ}$ prémolaire & 0,17 & 0,20 \\
Moyenne & 0,144 & 0,418 \\
\hline
\end{tabular}

Tableau 3 : IHA (Individual Hypoplastic Area) pour chaque patient.

IHA (Individual Hypoplastic Area) for each patient.

médecine

buccale

chirurgie

buccale

VOL. $14, \mathrm{~N}^{\circ} 1$

2008

page 25

\begin{tabular}{ccc} 
Patient & IHA \\
\hline 1 & 0,11 \\
2 & 0,22 \\
3 & 0,11
\end{tabular}

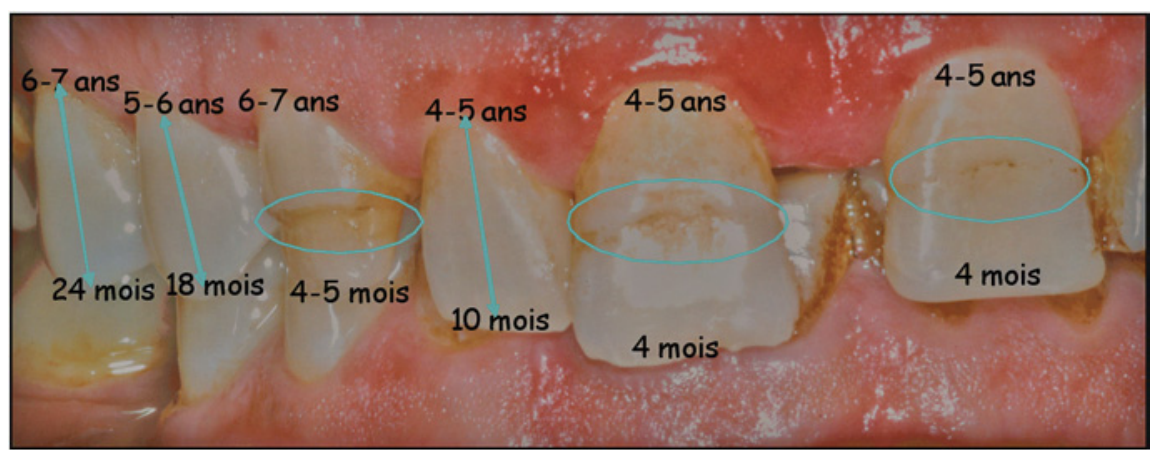

Figure 11 : Faces vestibulaires maxillaires des incisives centrales et de la canine atteintes d'hypoplasie chez le patient 1 ; l'incisive latérale et les prémolaires sont indemnes. (Dates de minéralisation de couronnes d'après Lautrou [21). Buccal view showing hypoplasia on medial incisives and canine (patient 1). The others teeth are not damaged. (Dates of calcification according Lautrou [21]). 
médecine

buccale

chirurgie

buccale

VOL. $14, \mathrm{~N}^{\circ} 1$ 2008

page 26 spatio-temporelle. Cependant, cette théorie est difficile à retenir pour ces patients car dans un même champ morphogénétique, on retrouve une dent atteinte et une dent indemne (Fig. 11). L'hypothèse d'une théorie autoimmune expliquant l'apparition d'hypoplasies peut également être prise en compte : en effet, les mutations du gène AIRE, à l'origine des manifestations autoimmunes du syndrome APECED, aboutissent à des cellules autoréactives qui peuvent endommager l'organe de l'émail par une attaque autoimmune. Le dommage direct des organes amélaires par un épisode autoimmun serait donc une cause probable d'hypoplasie [3]. Actuellement, l'étiologie autoimmune des altérations de l'émail est de plus en plus évoquée. Cette hypothèse est retenue pour les patients allergiques au gluten [15-19]. Wierink et coll. [15] ont évalué les hypoplasies amélaires chez les patients ayant une symptomatologie de malabsorption intestinale ou une maladie cœliaque confirmée par une biopsie positive ou infirmée par une absence de résultats lors de la biopsie. Les patients ayant une maladie cœliaque caractérisée présentent plus d'hypoplasies, ce qui serait en faveur d'une étiologie autoimmune. De même, Mariani et coll. [19] ont mis en évidence que les patients présentant l'antigène HLADR3 avaient un risque augmenté de lésions amélaires alors que ceux qui souffraient de malabsorption - potentiellement une absorption diminuée en calcium - présentaient moins d'altérations de l'émail. L'hypothèse de la perturbation du métabolisme phosphocalcique est également une étiologie classiquement proposée [20]. Cependant dans le syndrome APECED, il existe des hypoplasies sans que I'hypoparathyroïdie soit diagnostiquée, comme pour le patient 3 . De même, pour le patient 1 , le diagnostic de l'hypoparathyroïdie ayant été fait à l'adolescence (16 ans), on peut donc supposer que I'hypoparathyroïdie ne s'était pas encore développée pendant l'enfance, particulièrement pendant la période de minéralisation des couronnes des incisives qui s'étale de 3 mois à $4-5$ ans. Pour le patient 2, les hypoplasies pourraient être liées à l'atteinte parathyroïdienne précoce avec probablement un début d'altération du métabolisme phosphocalcique bien avant le diagnostic d'hypoparathyroïdie, à savoir pendant les périodes de calcification dentaire. Cependant, l'atteinte des couronnes des deuxièmes prémolaires mandibulaires et des dents de sagesse réfutent cette hypothèse puisqu'elles se minéralisent lorsque l'hypoparathyroïdie est traitée. En fait, bien que la survenue de l'hypoplasie amélaire ne soit pas initiée par l'hypoparathyroïdie, celle-ci pourrait potentialiser l'atteinte amélaire. D'autres études, avec un plus grand nombre de patients, des précisions sur la date d'apparition de l'hypoparathyroïdie et des évaluations détaillées des hypoplasies amélaires de toutes les couronnes des dents permanentes seraient nécessaires.

A l'image de la société Nord-Américaine de Gastroentérologie pédiatrique, Hépatologie et Nutrition qui considère les hypoplasies de l'émail comme un symptôme de la maladie cœliaque, les hypoplasies rencontrées dans le syndrome APECED pourraient constituer un des éléments conduisant au diagnostic de cette maladie, lorsque la « triade caractéristique » n'est pas encore présente.

\section{CONCLUSION}

Au même titre que dans la maladie cœliaque, les hypoplasies dentaires font partie des signes cliniques du syndrome l'APECED. La variabilité dans l'apparition des symptômes retarde parfois le diagnostic, et la mise en évidence d'hypoplasies amélaires caractérisées permettrait dans certains cas un diagnostic plus précoce. De surcroît, l'observation de la localisation des hypoplasies des patients étudiés contribue à étayer une théorie autoimmune des hypoplasies amélaires, comme dans la maladie cœliaque. En effet, elles seraient plutôt la conséquence d'une pathologie autoimmune que secondaires à un déficit endocrinien ou à une altération du métabolisme phosphocalcique. Cette étude montre que l'odontologiste, outre son rôle primordial dans la détection de nouveaux cas, doit être intégré dans l'équipe médicale pluridisciplinaire qui assure la prise en charge des syndromes APECED.

\section{REMERCIEMENTS}

Les auteurs remercient les familles et les patients qui ont participé à cette étude pour leur coopération ainsi que les médecins (Pr Warnet, Dr Crosnier et $\mathrm{Dr}$ Zenaty) qui les suivent. Ils remercient aussi le $\mathrm{Dr}$ Collin pour ses remarques avisées. 


\section{RÉFÉRENCES}

1 - Ahonen P, Myllarniemi S, Sipila I, Perheentupa J. Clinical variation of autoimmune polyendocrinopathy-candidiasis-ectodermal dystrophy (APECED) in a series of 68 patients. N Engl J Med 1990 ; 322 : 1829-36.

2 - Betterle C, Greggio NA, Volpato M. Autoimmune polyglandular syndrome type 1. J Clin Endocrinol Metab 1998 ; 83 : 1049-55.

3 - Perheentupa J. APS-I / APECED: the clinical disease and therapy. Endocrinol Metab Clin N Am 2002 ; 31 : 295-320.

4 - Shapiro MS, Zamir R, Weiss E, Radnay J, Schenkman $\mathrm{L}$. The polyglandular deficiency syndrome: a new variant in Persian Jews. J Endocrino Invest $1987 ; 10: 1-7$.

5 - Perniola R, Tamborrino G, Marsigliante S, De Rinaldis C. Assessment of enamel hypoplasia in autoimmune polyendocrinopathy-candidiasis-ectodermal dystrophy (APECED). J Oral Pathol Med 1998 ; 27 : 278-82.

6 - Lukinmaa PL, Waltimo J, Pirinen S. Microanatomy of the dental enamel in autoimmune polyendocrinopathycandidiasis-ectodermal dystrophy (APECED): report of three cases. J Craniofac Genet Dev Biol 1996 ; 16 : 174-81.

7 - Fédération Dentaire Internationale. A review of the developmental defects of dental enamel index (DDE Index). Int Dent J 1992 ; 42 : 411-26.

8 - Elcock C, Lath DL, Luty JD, Gallagher M, Abdellatif A, Brook $A$. The new enamel defects index: testing and expansion. Eur J Oral Sci 2006 ; 114 : 35-8.

9 - Blakey ML, Armelagos GJ. Comment on "hypoplastic area method for analysing enamel hypoplasia." Am J Phys Anthropol 1997 ; 102 : 295-6.

10 - Ensor BE, Irish JD. Reply to Blakey and Armelagos, with additional remarks on the hypoplastic area method. Am J Phys Anthropol 1997 ; 102 : 296-9.
11 - Hutchinson DL, Larsen CS. Determination of stress episode duration from linear enamel hypoplasia: a case study from St. Catherine Island, Georgia. Hum Biol $1988 ; 60: 93-110$.

12 - Ensor BE, Irish JD. Hypoplastic area method for analyzing dental enamel hypoplasia. Am J Phys Anthropol $1995 ; 98: 507-17$.

13 - Azogui-Lévy S, Boy-Lefevre ML. La santé bucco-dentaire en France. Actualité et dossier en santé publique $2005 ; 51: 4-8$.

14 - Thomas BL, Sharpe PT. Patterning of the murine dentition by homeobox gene. Eur J Oral Sci $1998 ; 106$ (suppl.1) : 48-54.

15 - Wierink C, Van Diermen D, Aartman I, Heymans H. Dental enamel defects in children with coeliac disease. Int J Paed Dent $2007 ; 17$ :163-8.

16 - Aine L. Coeliac-type permanent-tooth enamel defects. Ann Med 1996 ; 28 : 9-12.

17 - Aine L, Mäki M, Collin P, Keyriläinen O. Dental enamel defects in coeliac disease. J Oral Pathol Med 1990 ; $19: 241-5$

18 - Mäki M, Aine L, Lipsanen V, Koskimies S. Dental enamel defects in first-degree relatives of celiac patients. Lancet $1991 ; 337:$ 763-4.

19 - Mariani P, Mazzili MC, Margutti G. Coeliac disease, enamel defects and HLA typing. Acta Paediatr 1994 ; $83: 1272-5$.

20 - Bailleul-Forestier I. Vitamine D et odontologie pédiatrique. Odontostomatol Pediat 2003 ; 10 : 145-9.

21 - Lautrou A. Abrégé d'anatomie dentaire. Masson, Paris 1986. \begin{tabular}{l}
$\begin{array}{l}\text { médecine } \\
\text { buccale } \\
\text { chirurgie } \\
\text { buccale }\end{array}$ \\
\hline VOL. $14, N^{\circ} 1$ \\
2008
\end{tabular}

page 27 